University of Wollongong

Research Online

Faculty of Engineering and Information

Faculty of Engineering and Information

Sciences - Papers: Part B

Sciences

2018

A series-form solution for pricing variance and volatility swaps with stochastic volatility and stochastic interest rate

Xinjiang He

University of Wollongong, xinjiang@uow.edu.au

Song-Ping Zhu

University of Wollongong, spz@uow.edu.au

Follow this and additional works at: https://ro.uow.edu.au/eispapers1

Part of the Engineering Commons, and the Science and Technology Studies Commons

Research Online is the open access institutional repository for the University of Wollongong. For further information contact the UOW Library: research-pubs@uow.edu.au 


\title{
A series-form solution for pricing variance and volatility swaps with stochastic volatility and stochastic interest rate
}

\author{
Abstract \\ In this paper, we present analytical pricing formulae for variance and volatility swaps, when both of the \\ volatility and interest rate are assumed to be stochastic and follow a CIR (Cox-Ingersoll-Ross) process, \\ forming a Heston-CIR hybrid model. The solutions are written in a series form with a theoretical proof of \\ their convergence, ensuring the accuracy of the determined swap prices. The application of the formulae \\ in practice is also demonstrated through the designed numerical experiments. \\ Disciplines \\ Engineering | Science and Technology Studies

\section{Publication Details} \\ He, X. \& Zhu, S. (2018). A series-form solution for pricing variance and volatility swaps with stochastic \\ volatility and stochastic interest rate. Computers and Mathematics with Applications, 76 (9), 2223-2234.
}




\title{
A series-form solution for pricing variance and volatility swaps with stochastic volatility and stochastic interest rate
}

\author{
Xin-Jiang $\mathrm{He}^{*}$, Song-Ping Zhu \\ School of Mathematics and Applied Statistics, University of Wollongong, NSW 2522, Australia
}

\section{A R T I C L E I N F O}

\section{Article history:}

Received 11 March 2018

Received in revised form 11 July 2018

Accepted 11 August 2018

Available online 28 August 2018

\section{Keywords:}

Analytical pricing formulae

Variance and volatility swaps

Heston-CIR hybrid model

Series solution

Convergence

\begin{abstract}
A B S T R A C T
In this paper, we present analytical pricing formulae for variance and volatility swaps, when both of the volatility and interest rate are assumed to be stochastic and follow a CIR (CoxIngersoll-Ross) process, forming a Heston-CIR hybrid model. The solutions are written in a series form with a theoretical proof of their convergence, ensuring the accuracy of the determined swap prices. The application of the formulae in practice is also demonstrated through the designed numerical experiments.
\end{abstract}

(C) 2018 Elsevier Ltd. All rights reserved.

\section{Introduction}

Due to the popularity of financial markets, effectively managing financial risk caused by the high trading volumes of different kinds of financial derivatives is becoming increasingly demanding, which prompts the development of volatility derivatives that are able to provide direct exposure towards the assets volatility without investing the assets themselves. Among them, variance and volatility swaps, whose values depend on the future realized variance and volatility, respectively, are two kinds of the most popular volatility derivatives, and thus how to accurately and efficiently determine their prices has attracted a lot of research interest.

In the literature, one of the most widely adopted assumptions in pricing variance and volatility swaps is that the realized variance or volatility is continuously sampled. For example, Carr \& Lee [1,2] presented general model independent results, while a number of authors have worked on this area by incorporating stochastic volatility into pricing models [3-6]. Although appealing, such kind of assumption is at odds with the market practice, where the realized variance or volatility is discretely sampled in real markets, which can lead to mis-valuation of variance and volatility swaps and has been pointed out by a number of authors $[7,8]$.

In order to properly reflect the discrete sampling effect and be closer to financial reality, the recent trend is to work on the valuation of discretely-sampled variance and volatility swaps. In general, dealing with financial derivatives based on discretely-sampled data as their inputs is usually less straightforward than dealing with continuously-sampled derivatives as mentioned in [9-11]; results on the pricing of discretely-sampled variance and volatility swaps are usually presented based on different models. For example, using the finite difference method, discretely sampled variance swaps are priced under a local volatility model by Little \& Pant [7] with a high-order of accuracy via a dimension-reduction approach, which is extended by Zhu \& Lian [12] in deriving a closed-form pricing formula for variance swaps under the Heston model [13]. The analytical solution for volatility swaps under the Heston model has also been derived in [14], and analytical as well as

\footnotetext{
* Corresponding author.

E-mail address: xinjiang@uow.edu.au (X.-J. He).
} 
asymptotic results for discrete-sampled variance swaps are presented under three different stochastic volatility models [15]. It needs to be pointed out that stochastic volatility models alone are not adequate to reflect market observations, and thus other potential factors are introduced, in order to provide better fit to market data. For example, jumps have also been introduced in the underlying price in analyzing their effect on variance and volatility swap prices [16]. Moreover, Markov modulated models are also attracting attention due to empirical evidence showing the existence of regime-switching in real markets [17], and a number of different authors have worked on derivative pricing problems under these models [18-20]. Belonging to this category, a regime-switching Heston model was adopted in [8] for the analytical evaluation of variance and volatility swaps. A survey of the literature on the volatility derivatives in real markets can be found in [21].

In this paper, we adopt the Heston-CIR hybrid model, combining the Heston stochastic volatility model and CIR (CoxIngersoll-Ross) stochastic interest rate model. In fact, stochastic interest rate models have already been widely adopted in pricing financial derivatives. For example, the valuation problem of European options under the correlated Heston stochastic volatility and CIR or Hull-White [22] stochastic interest rate models is considered by Grzelak \& Oosterlee [23], while American option pricing under a general hybrid stochastic volatility and stochastic interest rate model is discussed in [24] with a short-maturity asymptotic expansion. Kim et al. [25] went even further and showed that adding stochastic interest rates into a stochastic volatility model could give better results compared with the constant interest rate case in any maturity. It needs to be pointed out that although Cao et al. [26] have already worked on the determination of variance swap prices under this model, their formula is not analytical since it involves solving some ODEs (ordinary differential equations) when computing the price of any variance swap, which may lead to inaccuracy problems if numerical methods are resorted to when finding solutions to these ODEs. In order to overcome this disadvantage, we present analytical pricing formulae for variance and volatility swaps, based on the derived forward characteristic function. This particular solution is actually in a series form, accompanied by a radius of convergence, ensuring the safety of its application in real markets. To demonstrate the accuracy and efficiency of the new formulae, numerical experiments are carried out to show the speed of convergence, followed by a comparison of swap prices calculated with our formulae and those obtained from Monte Carlo simulation. The influence of introducing stochastic interest rate into the Heston model is also studied.

The rest of the paper is organized as follows. In Section 2, we introduce the hybrid stochastic volatility and stochastic interest rate model. In Section 3, general formulae for variance and volatility swap prices involving an unknown forward characteristic function are presented, and the unknown function is then figured out through inverse Fourier transform. Numerical examples and discussions are presented in Section 4, followed by some concluding remarks given in the last section.

\section{The Heston-CIR hybrid model}

Despite the popularity of the Black-Scholes model [27], a lot of empirical evidence has already demonstrated that some simple assumptions made in this particular model are not consistent with real market observations, including the constant volatility assumption [28] and the constant interest rate assumption [29]. Therefore, large amount of research interest has been led into modifying the classical Black-Scholes model, by incorporating stochastic or local volatility, stochastic interest rate and jump diffusion [30-32]. Among the category of stochastic volatility, the Heston model with the volatility following the CIR process is very popular among academic researchers and market practitioners because the model itself not only satisfies a number of basic properties, such as the non-negative property and the mean-reverting property being consistent with real market data, but also provides analytical pricing formulae for some popular financial derivatives [12-14]. Due to similar reasons, the CIR process is also widely used to model the stochastic interest rate models [33]. In this case, what we adopt here is a combination of the Heston stochastic volatility model and the CIR stochastic interest rate model. The formulated Heston-CIR hybrid model under a risk-neutral measure $\mathbb{Q}$ can be expressed as

$$
\left[\begin{array}{c}
d S_{t} \\
S_{t} \\
d v_{t} \\
d r_{t}
\end{array}\right]=\mu^{Q} d t+\Sigma \times C \times\left[\begin{array}{l}
d W_{1, t} \\
d W_{2, t} \\
d W_{3, t}
\end{array}\right],
$$

with $S_{t}, v_{t}$ and $r_{t}$ being the underlying price, volatility and risk-free interest rate at time $t$, respectively. $W_{1, t}, W_{2, t}$ and $W_{3, t}$ are three standard Brownian motions that are independent with each other. The drift term $\mu^{Q}$ and the volatility term $\Sigma$ are defined as

$$
\mu^{Q}=\left[\begin{array}{c}
r_{t} \\
k\left(\theta-v_{t}\right) \\
\alpha\left(\beta-r_{t}\right)
\end{array}\right], \quad \Sigma=\left[\begin{array}{ccc}
\sqrt{v_{t}} & 0 & 0 \\
0 & \sigma \sqrt{v_{t}} & 0 \\
0 & 0 & \eta \sqrt{r_{t}}
\end{array}\right],
$$

and $C$ is given as 
such that the correlation matrix is

$$
C C^{T}=\left[\begin{array}{lll}
1 & \rho & 0 \\
\rho & 1 & 0 \\
0 & 0 & 1
\end{array}\right] .
$$

With the dynamics of the underlying price, volatility and interest rate following the Heston-CIR hybrid model introduced above, we are going to present the derivation process for the analytical expression of variance and volatility swap prices, details of which are shown in the next section.

\section{Valuation of variance and volatility swaps}

\subsection{General pricing formulae}

Before we present the details, it is necessary to make it clear what is a "price" for a variance or volatility swap contract. Unlike many other financial derivatives, whose prices are defined as the values of these contracts, variance and volatility swaps are special kinds of forward contracts, and their prices are referred to as the delivery price specified in the contracts. In other words, what we need to determine is the delivery price that the long position of variance or volatility swaps needs to pay at expiry, in exchange of receiving the floating amount of the realized variance or volatility within the time period of the contract.

If we assume $K_{v a r}$ and $K_{v o l}$ are respectively the delivery price of a variance and volatility swap contract that need to be determined, and use $R V_{v a r}$ and $R V_{v o l}$ to represent the annualized realized variance and volatility respectively, the values of the two contracts, $V_{v a r}$ and $V_{v o l}$, will have the expression of

$$
V_{v a r}=E^{Q}\left[e^{-\int_{0}^{T} r_{t} d t}\left(R V_{v a r}-K_{v a r}\right) L \mid S_{0}, v_{0}, r_{0}\right], \quad V_{v o l}=E^{Q}\left[e^{-\int_{0}^{T} r_{t} d t}\left(R V_{v o l}-K_{v o l}\right) L \mid S_{0}, v_{0}, r_{0}\right],
$$

where $L$ is the notional amount specified in the contracts. Given that the value of any forward contract should equal to zero when it is entered since it should be fair to both parties, we can obtain the following two identities

$$
E^{Q}\left[e^{-\int_{0}^{T} r_{t} d t}\left(R V_{v a r}-K_{v a r}\right) L \mid S_{0}, v_{0}, r_{0}\right]=0, \quad E^{Q}\left[e^{-\int_{0}^{T} r_{t} d t}\left(R V_{v o l}-K_{v o l}\right) L \mid S_{0}, v_{0}, r_{0}\right]=0,
$$

which implies that the first step is to evaluate the two expectations involved, depending on the definitions of the realized variance and volatility. What we choose here is one of the most widely adopted definitions in the literature $[4,8,12]$, where $R V_{v a r}$ and $R V_{v o l}$ are respectively represented as

$$
\begin{aligned}
& R V_{\text {var }}=\frac{100^{2}}{T} \sum_{i=1}^{N}\left(\frac{S_{t_{i}}-S_{t_{i-1}}}{S_{t_{i-1}}}\right)^{2}, \\
& R V_{\text {vol }}=100 \sqrt{\frac{\pi}{2 N T}} \sum_{i=1}^{N}\left|\frac{S_{t_{i}}-S_{t_{i-1}}}{S_{t_{i-1}}}\right|,
\end{aligned}
$$

with $T$ being the expiry time and the time period $[0, T]$ being uniformly discretized into $N$ small periods $\left[t_{i-1}, t_{i}\right]$ for $i=1,2, \ldots, N$. Although our task of evaluating the two expectations is very clear, direct calculation is extremely difficult since it requires computing the expectations of the product of two dependent random variables. Fortunately, with the help of the forward measure $\mathbb{Q}^{T}$, the expectations can be reformulated as

$$
\begin{aligned}
E^{Q}\left[e^{-\int_{0}^{T} r_{t} d t}\left(R V_{v a r}-K_{v a r}\right) L \mid S_{0}, v_{0}, r_{0}\right] & =P(r, 0, T) E^{Q^{T}}\left[\left(R V_{v a r}-K_{v a r}\right) L \mid S_{0}, v_{0}, r_{0}\right], \\
E^{Q}\left[e^{-\int_{0}^{T} r_{t} d t}\left(R V_{v o l}-K_{v o l}\right) L \mid S_{0}, v_{0}, r_{0}\right] & =P(r, 0, T) E^{Q^{T}}\left[\left(R V_{v o l}-K_{v o l}\right) L \mid S_{0}, v_{0}, r_{0}\right],
\end{aligned}
$$

where $P(r, t, T)=E^{Q}\left[e^{-\int_{t}^{T} r_{s} d s} \mid r_{t}\right]$ is the price of a $T$-forward zero-coupon bond at the current time $t$. Therefore, we can finally arrive at a general formula for the price of a variance and volatility swap

$$
\begin{aligned}
& K_{v a r}=E^{Q^{T}}\left[R V_{v a r}\right]=\frac{100^{2}}{T} \sum_{i=1}^{N} E^{Q^{T}}\left[\left(\frac{S_{t_{i}}-S_{t_{i-1}}}{S_{t_{i-1}}}\right)^{2} \mid S_{0}, v_{0}, r_{0}\right], \\
& K_{v o l}=E^{Q^{T}}\left[R V_{v o l}\right]=100 \sqrt{\frac{\pi}{2 N T}} \sum_{i=1}^{N} E^{Q^{T}}\left[\left|\frac{S_{i}-S_{t_{i-1}}}{S_{t_{i-1}}}\right| S_{0}, v_{0}, r_{0}\right] .
\end{aligned}
$$

Obviously, the remaining task is to work out the $2 \mathrm{~N}$ expectations presented in the above formulae. With the transforma- 
the delivery price of a variance swap can be simplified as

$$
\begin{aligned}
K_{v a r} & =\frac{100^{2}}{T} \sum_{i=1}^{N} E^{Q^{T}}\left[\left(\frac{S_{t_{i}}-S_{t_{i-1}}}{S_{t_{i-1}}}\right)^{2} \mid S_{0}, v_{0}, r_{0}\right] \\
& =\frac{100^{2}}{T} \sum_{i=1}^{N} E^{Q^{T}}\left[\left(e^{y_{t_{i-1}, t_{i}}}-1\right)^{2} \mid S_{0}, v_{0}, r_{0}\right] \\
& =\frac{100^{2}}{T} \sum_{i=1}^{N} E^{Q^{T}}\left[e^{2 y_{t_{i-1}}, t_{i}}-2 e^{y_{t_{i-1}}, t_{i}}+1 \mid S_{0}, v_{0}, r_{0}\right] .
\end{aligned}
$$

If we denote $f\left(\phi, t, T ; S_{0}, v_{0}, r_{0}\right)$ as the forward characteristic function of the log-returns of the underlying price under the forward measure $\mathbb{Q}^{T}$, we have

$$
f\left(\phi, t, T ; S_{0}, v_{0}, r_{0}\right)=E^{Q^{T}}\left[e^{j \phi y_{t, T}} \mid S_{0}, v_{0}, r_{0}\right],
$$

and thus we can directly obtain

$$
K_{\text {var }}=\frac{100^{2}}{T} \sum_{i=1}^{N}\left[f\left(-2 j, t_{-1}, t_{i} ; S_{0}, v_{0}, r_{0}\right)-2 f\left(-j, t_{i-1}, t_{i} ; S_{0}, v_{0}, r_{0}\right)+1\right],
$$

With the same transformation shown in Eq. (3.1), and the forward density function of $y_{t_{i-1}, t_{i}}$ being denoted by $p\left(y_{t_{i-1}, t_{i}}\right)$, the expectations contained in the volatility swap pricing formulae can be expressed as

$$
\begin{aligned}
E^{Q^{T}}\left[\left|\frac{S_{t_{i}}-S_{t_{i-1}}}{S_{t_{i-1}}}\right| \mid S_{0}, v_{0}, r_{0}\right] & =\int_{0}^{+\infty}\left(e^{y_{t_{i-1}, t_{i}}}-1\right) p\left(y_{t_{i-1}, t_{i}}\right) d y_{t_{i-1}, t_{i}}+\int_{-\infty}^{0}\left(1-e^{y_{t_{i-1}, t_{i}}}\right) p\left(y_{t_{i-1}, t_{i}}\right) d y_{t_{i-1}, t_{i}} \\
& =-\int_{0}^{+\infty} p\left(y_{t_{i-1}, t_{i}}\right) d y_{t_{i-1}, t_{i}}+\int_{-\infty}^{0} p\left(y_{t_{i-1}, t_{i}}\right) d y_{t_{i-1}, t_{i}} \\
& +\int_{0}^{+\infty} e^{y y_{i-1}, t_{i}} p\left(y_{t_{i-1}, t_{i}}\right) d y_{t_{i-1}, t_{i}}-\int_{-\infty}^{0} e^{y t_{i-1}, t_{i}} p\left(y_{t_{i-1}, t_{i}}\right) d y_{t_{i-1}, t_{i}} .
\end{aligned}
$$

The first two integrals can be easily evaluated by making use of the relationship between the density function and characteristic function

$$
\int_{0}^{+\infty} p\left(y_{t_{i-1}, t_{i}}\right) d y_{t_{i-1}, t_{i}}=\frac{1}{2}+\frac{1}{\pi} \int_{0}^{+\infty} R E\left[\frac{f\left(\phi, t_{i-1}, t_{i} ; S_{0}, v_{0}, r_{0}\right)}{j \phi}\right] d \phi
$$

where $R E[\cdot]$ represents taking the real part. On the other hand, setting $\phi=j$ into Eq. (3.2) yields

$$
\int_{-\infty}^{+\infty} e^{y_{t_{-1}, t_{i}}} p\left(y_{t_{i-1}, t_{i}}\right) d y_{t_{i-1}, t_{i}}=f\left(-j, t_{i-1}, t_{i} ; S_{0}, v_{0}, r_{0}\right)
$$

which implies that $\frac{e^{y_{t_{i-1}, t_{i}}} p\left(y_{t_{i-1}, t_{i}}\right)}{f\left(-j, t_{i-1}, t_{i} S_{0}, v_{0}, r_{0}\right)}$ is actually a density function of another random variable, with its characteristic function derived through taking the Fourier transform of the density

$$
\begin{aligned}
\bar{f}\left(\phi, t_{i-1}, t_{i} ; S_{0}, v_{0}, r_{0}\right) & =\int_{-\infty}^{+\infty} e^{\phi \phi y_{t_{i-1}, t_{i}}} \frac{e^{y_{t_{i-1}, t_{i}} p\left(y_{t_{-1}, t_{i}}\right)}}{f\left(-j, t_{i-1}, t_{i} ; S_{0}, v_{0}, r_{0}\right)} d y_{t_{i-1}, t_{i}} \\
& =\frac{f\left(\phi-j, t_{i-1}, t_{i} ; S_{0}, v_{0}, r_{0}\right)}{f\left(-j, t_{i-1}, t_{i} ; S_{0}, v_{0}, r_{0}\right)} .
\end{aligned}
$$

In this case, we can further obtain

$$
\int_{0}^{+\infty} \frac{e^{y_{t_{i-1}, t_{i}}} p\left(y_{t_{i-1}, t_{i}}\right)}{f\left(-j, t_{i-1}, t_{i} ; S_{0}, v_{0}, r_{0}\right)} d y_{t_{i-1}, t_{i}}=\frac{1}{2}+\frac{1}{\pi} \int_{0}^{+\infty} R E\left[\frac{f\left(\phi-j, t_{i-1}, t_{i} ; S_{0}, v_{0}, r_{0}\right)}{j \phi \cdot f\left(-j, t_{i-1}, t_{i} ; S_{0}, v_{0}, r_{0}\right)}\right] d \phi,
$$

which leads to 
Therefore, we finally arrive at the general formula for the delivery price of a volatility swap

$$
\begin{aligned}
K_{v o l} & =100 \sqrt{\frac{\pi}{2 N T}} \sum_{i=1}^{N} E^{Q^{T}}\left[\left|\frac{S_{t_{i}}-S_{t_{i-1}}}{S_{t_{i-1}}}\right| \mid S_{0}, v_{0}, r_{0}\right] \\
& =100 \sqrt{\frac{2}{\pi N T}} \int_{0}^{+\infty} \sum_{i=1}^{N} R E\left[\frac{f\left(\phi-j, t_{i-1}, t_{i} ; S_{0}, v_{0}, r_{0}\right)-f\left(\phi, t_{i-1}, t_{i} S_{0}, v_{0}, r_{0}\right)}{j \phi}\right] d \phi .
\end{aligned}
$$

From the expression of the formulae, (3.3) and (3.10), it is not difficult to find that the only unknown term involved is the forward characteristic function, and the pricing formulae can be directly applied after it is successfully worked out. Thus, the details of analytically deriving the forward characteristic function are presented in the next subsection.

\subsection{Forward characteristic function}

In order to derive the forward characteristic function, we start with its definition in Eq. (3.2), from which it is clear that our task is to evaluate the expectation $E^{Q^{T}}\left[e^{j \phi y_{t, T}} \mid S_{0}, v_{0}, r_{0}\right]$. It should be noted that this target expectation is under the $T$-forward measure $\mathbb{Q}^{T}$, while the dynamics of the underlying price, volatility and interest rate we currently have are under the measure $\mathbb{Q}$. Thus, the first step is to try to derive the dynamics under $\mathbb{Q}^{T}$.

In fact, the numeraire under $\mathbb{Q}$ is $N_{1, t}=e^{\int_{0}^{t} r(s) d s}$, and the numeraire under $\mathbb{Q}^{T}$ is $N_{2, t}=P(r, t, T)$. Applying the RadonNikodym derivative to the two numeraires can yield the model dynamics under the $T$-forward measure $\mathbb{Q}^{T}$ after measure transform ${ }^{1}$

$$
\left[\begin{array}{c}
d S_{t} \\
S_{t} \\
d v_{t} \\
d r_{t}
\end{array}\right]=\left[\begin{array}{c}
r_{t} \\
k\left(\theta-v_{t}\right) \\
\alpha \beta-\left[\alpha+B(t, T) \eta^{2}\right] r_{t}
\end{array}\right] d t+\Sigma \times C \times\left[\begin{array}{c}
d W_{1, t}^{Q^{T}} \\
d W_{2, t}^{Q^{T}} \\
d W_{3, t}^{Q^{T}}
\end{array}\right],
$$

where

$$
B(t, T)=\frac{2\left(e^{m(T-t)}-1\right)}{2 m+(\alpha+m)\left(e^{m(T-t)}-1\right)},
$$

with $m=\sqrt{\alpha^{2}+2 \eta^{2}}$.

Obviously, the next step of our approach is to calculate the expectation in Eq. (3.2) under the $T$-forward measure $\mathbb{Q}^{T}$. However, $y_{t, T}$ involves two random variables, $S_{t}$ and $S_{T}$, which means that the direct computation is very complicated, making us to seek for alternative methods. In fact, the forward characteristic function can be transformed as

$$
\begin{aligned}
f\left(\phi, t, T ; S_{0}, v_{0}, r_{0}\right) & =E^{Q^{T}}\left[e^{j \phi y_{t, T}} \mid S_{0}, v_{0}, r_{0}\right] \\
& =E^{Q^{T}}\left\{E^{Q^{T}}\left[e^{j \phi y_{t, T}} \mid S_{t}, v_{t}, r_{t}\right] \mid S_{0}, v_{0}, r_{0}\right\},
\end{aligned}
$$

in which case both of the inner and outer expectation involve only one random variable since $S_{t}$ in the inner expectation is known at time $t$ and it is also the only random variable explicitly shown in the outer expectation. As a result, our solution procedure is divided into two stages, with each stage dealing with one expectation. The result of the inner expectation is presented in the following proposition.

Proposition 3.1. If the underlying price follows the dynamics specified in Eq. (3.11), the inner expectation in (3.13) can be derived as

$$
E^{Q^{T}}\left[e^{j \phi y_{t, T}} \mid S_{t}, v_{t}, r_{t}\right]=e^{C(\phi ; \tau)+D(\phi ; \tau) v_{t}+E(\phi ; \tau) r},
$$

where

$$
\begin{aligned}
& D=\frac{d-(j \phi \rho \sigma-k)}{\sigma^{2}} \cdot \frac{1-e^{d \tau}}{1-g e^{d \tau}} \\
& E=-\frac{2 \sum_{n=0}^{+\infty}(n+1) \hat{a}_{n+1} \tau^{n}}{\eta^{2} \sum_{n=0}^{+\infty} \hat{a}_{n} \tau^{n}} \\
& C=\frac{k \theta}{\sigma^{2}}\left\{[d-(j \phi \rho \sigma-k)] \tau-2 \ln \left(\frac{1-g e^{d \tau}}{1-g}\right)-\alpha \beta \int_{0}^{\tau} \frac{2 \sum_{n=0}^{+\infty}(n+1) \hat{a}_{n+1} t^{n}}{\eta^{2} \sum_{n=0}^{+\infty} \hat{a}_{n} t^{n}} d t\right\}, \\
& d=\sqrt{(j \phi \rho \sigma-k)^{2}+\sigma^{2}\left(j \phi+\phi^{2}\right)}, g=\frac{(j \phi \rho \sigma-k)-d}{(i \alpha n \tau \text { l) । } d},
\end{aligned}
$$




$$
\begin{aligned}
\hat{a}_{n+2} & =-\frac{\hat{I}}{2 m(n+1)(n+2)}, n \geq 0, \hat{a}_{0}=1, \hat{a}_{1}=0, \\
\hat{I} & =2 \alpha m(n+1) \hat{a}_{n+1}+j \phi \eta^{2} m \hat{a}_{n}+(\alpha+m) \sum_{i=1}^{n}(n+2-i)(n+1-i) c_{i} \hat{a}_{n+2-i} \\
& +\left(\alpha^{2}+\alpha m+2 \eta^{2}\right) \sum_{i=1}^{n}(n+1-i) c_{i} \hat{a}_{n+1-i}+\frac{1}{2} j \phi \eta^{2}(\alpha+m) \sum_{i=1}^{n} c_{i} \hat{a}_{n-i},
\end{aligned}
$$

with $\tau=T-t$ and $c_{i}=\frac{m^{n}}{n !}$. Note: This series solution will always converge if

$$
\tau \leq \frac{1}{m} \sqrt{\left[\ln \left(\frac{m-\alpha}{m+\alpha}\right)\right]^{2}+\pi^{2}}
$$

The proof for this proposition is left in the Appendix.

With the derived inner expectation, the forward characteristic function can be simplified as

$$
f\left(\phi, t, T ; v_{0}, r_{0}\right)=E^{Q^{T}}\left[e^{C(\phi ; \tau)+D(\phi ; \tau) v_{t}+E(\phi ; \tau) r_{t}} \mid v_{0}, r_{0}\right]=e^{C(\phi ; \tau)} E^{Q^{T}}\left[e^{D(\phi ; \tau) v_{t}+E(\phi ; \tau) r_{t}} \mid v_{0}, r_{0}\right] .
$$

It should be remarked that $f\left(\phi, t, T ; v_{0}, r_{0}\right)$ is no longer dependent on the initial underlying asset price $S_{0}$ as a result of the disappearance of the underlying asset price in the expectation. In order to figure out the target expectation, we introduce a new function

$$
w\left(\phi, t, T ; v_{s}, r_{s}, s\right)=E^{Q^{T}}\left[e^{D(\phi ; \tau) v_{t}+E(\phi ; \tau) r_{t}} \mid v_{s}, r_{s}\right], s \in[0, t],
$$

which clearly satisfies the following PDE (partial differential equation)

$$
\frac{\partial w}{\partial s}+\frac{1}{2} \sigma^{2} v \frac{\partial^{2} w}{\partial v^{2}}+\frac{1}{2} \eta^{2} r \frac{\partial^{2} w}{\partial r^{2}}+k(\theta-v) \frac{\partial w}{\partial v}+\{\alpha \beta-[\alpha+B(s, T)] r\} \frac{\partial w}{\partial r}=0,
$$

with the termination condition $\left.w\right|_{s=t}=e^{D(\phi ; \tau) v t+E(\phi ; \tau) r_{t}}$. If $w\left(\phi, t, T ; v_{s}, r_{s}, s\right)$ is assumed to be in the form of

$$
w\left(\phi, t, T ; v_{s}, r_{s}, s\right)=e^{\bar{C}\left(\phi ; t_{s}\right)+\bar{D}\left(\phi ; t_{s}\right) v_{s}+\bar{E}\left(\phi ; t_{s}\right) r_{s}},
$$

with $t_{s}=t-s$, the above PDE can be transformed into three ODEs specified as

$$
\begin{aligned}
& \frac{d \bar{D}}{d t_{s}}=\frac{1}{2} \sigma^{2} \bar{D}^{2}-k \bar{D}, \\
& \frac{d \bar{E}}{d t_{s}}=\frac{1}{2} \eta^{2} \bar{E}^{2}-\left[\alpha+B(s, T) \eta^{2}\right] E, \\
& \frac{d \bar{C}}{d t_{s}}=k \theta \bar{D}+\alpha \beta \bar{E},
\end{aligned}
$$

with $C(\phi ; 0)=0, D(\phi ; 0)=D(\phi ; \tau)$ and $E(\phi ; 0)=E(\phi ; \tau)$. The ODEs governing $\bar{D}\left(\phi ; t_{s}\right)$ and $\bar{E}\left(\phi ; t_{s}\right)$ are both Bernoulli's equation, and $\bar{D}\left(\phi ; t_{s}\right)$ can be easily worked out with some algebraic calculations

$$
\bar{D}\left(\phi ; t_{s}\right)=\frac{2 k}{\sigma^{2}} \frac{1}{1-\left[1-\frac{2 k}{\sigma^{2} D(\phi ; \tau)}\right] e^{k t}} .
$$

In contrast, the derivation of $\bar{E}\left(\phi ; t_{s}\right)$ is a bit more complex due to the existence of a non-constant coefficient. In particular, if we denote

$$
\bar{B}\left(t_{s}, \tau\right)=B(s, T)=\frac{2\left(e^{m\left(t_{s}+\tau\right)}-1\right)}{2 m+(\alpha+m)\left(e^{m\left(t_{s}+\tau\right)}-1\right)},
$$

and make the transformation of $u\left(t_{s}\right)=\frac{1}{E\left(\phi ; t_{s}\right)}$, the ODE governing $\bar{E}\left(\phi ; t_{s}\right)$ is turned into

$$
u^{\prime}-\left[\alpha+\bar{B}\left(t_{s}, \tau\right) \eta^{2}\right] u=-\frac{1}{2} \eta^{2} .
$$

The resulting equation is actually a first-order ODE, the general solution to which can be expressed as

$$
u=\frac{\int_{0}^{t_{s}}-\frac{1}{2} \eta^{2} e^{-\int_{0}^{z} \alpha+\bar{B}(x, \tau) \eta^{2} d x} d z+c_{0}}{e^{-\int_{0}^{t_{s}} \alpha+\bar{B}(x, \tau) \eta^{2} d x}},
$$

where $c_{0}$ is a constant that needs to be determined through a certain initial condition. As a result, we can obtain 
the substitution of the initial condition into which leads to $c_{0}=\frac{1}{E(\phi ; \tau)}$. With the integral being analytically worked out as

$$
\int_{0}^{t_{s}} \bar{B}(x, \tau) d x=\frac{4}{(m-\alpha)(m+\alpha)} \ln \left\{\frac{2 m+(m+\alpha)\left[e^{m\left(\tau+t_{s}\right)}-1\right]}{2 m+(m+\alpha)\left(e^{m \tau}-1\right)}\right\}+\frac{2}{\alpha-m} t_{s},
$$

$\bar{E}\left(\phi ; t_{s}\right)$ can be alternatively expressed as

$$
\bar{E}\left(\phi ; t_{s}\right)=\frac{e^{-\left(\alpha+\frac{2 \eta^{2}}{\alpha-m}\right) t_{s}}\left\{\frac{2 m+(m+\alpha)\left[e^{m(\tau+t s)}-1\right]}{2 m+(m+\alpha)\left(e^{m \tau}-1\right)}\right\}^{-\frac{4 \eta^{2}}{(m-\alpha)(m+\alpha)}}}{-\frac{1}{2} \eta^{2} \int_{0}^{t_{s}} e^{-\left(\alpha+\frac{2 \eta^{2}}{\alpha-m}\right) x}\left\{\frac{2 m+(m+\alpha)\left[e^{m(\tau+x)}-1\right]}{2 m+(m+\alpha)\left(e^{m \tau}-1\right)}\right\}^{-\frac{4 \eta^{2}}{(m-\alpha)(m+\alpha)}} d x+\frac{1}{E(\phi ; \tau)}} .
$$

In this case, the derivation of $\bar{C}\left(\phi ; t_{s}\right)$ is quite straightforward by simply integrating on both sides of the governing ODE, which yields

$$
\bar{C}\left(\phi ; t_{s}\right)=\frac{2 k \theta}{\sigma^{2}}\left\{k t_{s}-\ln \left[1-\left(1-\frac{2 k}{\sigma^{2} D(\phi ; \tau)}\right) e^{k t_{s}}\right]+\ln \left(\frac{2 k}{\sigma^{2} D(\phi ; \tau)}\right)\right\}+\alpha \beta \int_{0}^{t_{s}} \bar{E}(\phi ; z) d z .
$$

Combining the results presented above, the forward characteristic function can be finally derived by setting $s=0$ (or $t_{s}=t$ )

$$
\begin{aligned}
f\left(\phi, t, T ; v_{0}, r_{0}\right) & =e^{C(\phi ; \tau)} w\left(\phi, t, T ; v_{0}, r_{0}, 0\right) \\
& =e^{C(\phi ; \tau)+\bar{C}(\phi ; t)+\bar{D}(\phi ; t) v_{0}+\bar{E}(\phi ; t) r_{0}} .
\end{aligned}
$$

Upon successfully presenting the analytical expression of the forward characteristic function, the delivery price of variance and volatility swaps can be determined through Eqs. (3.3) and (3.10), respectively. From the theoretical point of view, the newly derived formulae are mathematically appealing since the involved series solution is accompanied by a radius of convergence. However, from the practical point of view, the speed of convergence is also an important factor in the application of the formulae, especially in the recent trend of algorithmic trading. Thus, in the next section, numerical experiments are carried out to show whether the solution converges in a reasonably quick speed. In addition to the convergence, the accuracy of the formulae are also numerically checked by comparing our results and those obtained from Monte Carlo simulation, to ensure there are no algebraic errors, after which the influence of introducing stochastic interest rate into the Heston model is demonstrated.

\section{Numerical experiments and examples}

In this section, variance and volatility swap prices calculated with our newly derived formulae are used to perform some numerical analysis. Unless otherwise state in the following, the values of the parameters used are listed as follows. The initial risk-free interest rate $r_{0}$ and volatility $v_{0}$ are 0.03 and 0.05 , respectively, and the expiry time $T$ is 1 . The three parameters associated with the stochastic volatility process, the mean-reversion speed $k$, the mean-reversion level $\theta$, and the volatility of volatility $\sigma$, are set to be $10,0.2$, and 0.1 , respectively, while the three parameters related to the stochastic interest rate process, the mean-reversion speed $\alpha$, the mean-reversion level $\beta$, and the volatility of volatility $\eta$, takes the value of $2,0.05$ and 0.05 respectively. We use $\rho=-0.5$ as the correlation between the underlying price and volatility, and the sampling frequency $N$ is chosen to be 4 times per year. It should be remarked that as both formulae for variance and volatility swap prices use the same forward characteristic function, we will use variance swap prices as an example in showing their speed of convergence and accuracy.

Fig. 1 shows how our solution converges with different number of terms taken. In particular, what is shown in Fig. 1(a) is the absolute difference between $(n+1)$-term price and $n$-term price since it is widely accepted that the solution can be regarded as converged if such kind of difference becomes zero after the order of series reaches a critical point. Clearly, the absolute difference decreases very sharply to zero when we increase the number of terms used in the series, no matter what the time to expiry is, which means that our solution converges very rapidly and thus only a few terms can provide accurate results. To further demonstrate this, the 10-term and 11-term prices are plotted in Fig. 1(b) with respect to different sampling frequency, and it can be easily noticed that the two prices agree very well with each other, with the maximum absolute difference being less than $10^{-5}$. Thus, the 10 -term price is used to present numerical examples in the remaining of the section.

Having been aware of the speed of convergence for our formula, it is still necessary to check its accuracy so that the formula can be directly applied in real markets. Depicted in Fig. 2(a) is the comparison of variance swap prices calculated with our formula and those obtained from Monte Carlo simulation with different sampling frequency, and they are close to each other in a point-wise manner. To further identify the actual closeness of the two prices, the relative difference between variance swap prices obtained from the two approaches is shown in Fig. 2(b), and the maximum relative difference being less than $0.05 \%$ can of course demonstrate the accuracy of our formula. 


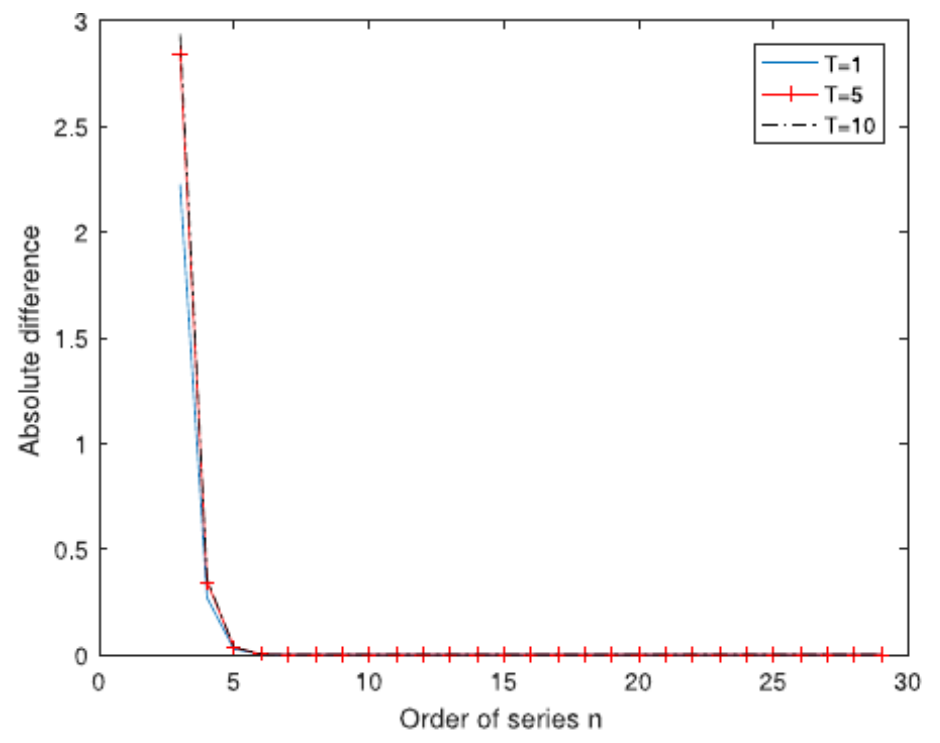

(a) Absolute difference between $(n+1)$-term and $n$-term variance swap price,

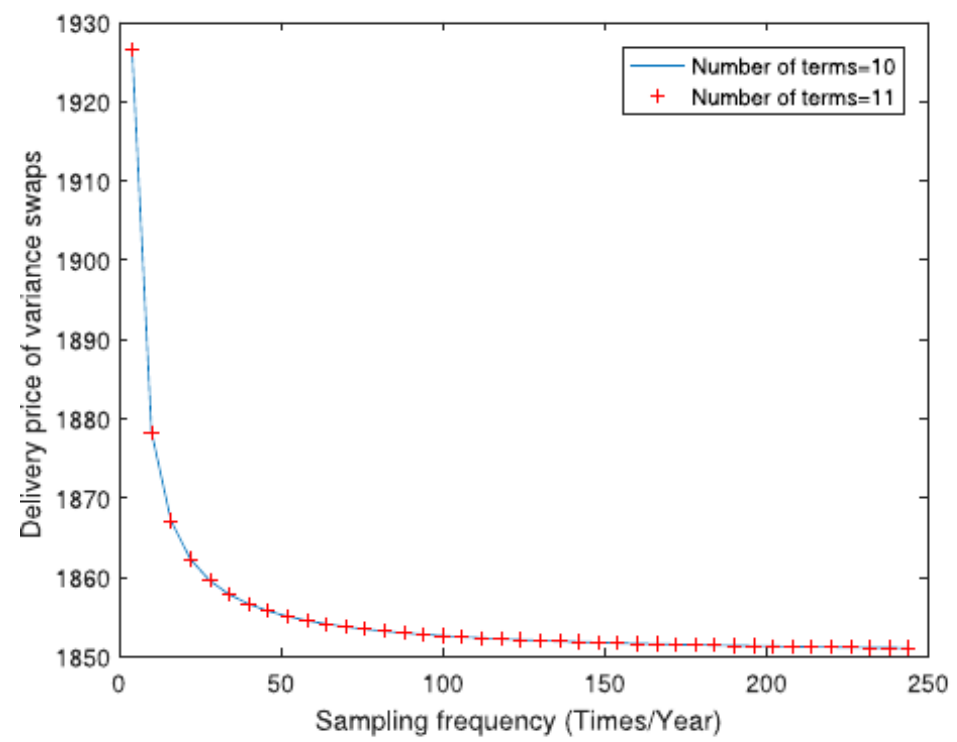

(b) The 10-term and 11-term variance swap prices.

Fig. 1. Speed of convergence for our formula.

find that our variance swap price is higher (lower) than the Heston price when the mean-reversion level is higher (lower) than the constant interest rate. This is because a higher mean-reversion level implies a higher interest rate in the long run, leading to higher variance swap price. A similar pattern can be observed in Fig. 3(b), except that the magnitude of the delivery price of volatility swaps is only about the square root of that of variance swaps, which is expected due to the definition of the realized variance and volatility. Obviously, the variation of the interest rate can contribute to a large difference in the variance and volatility swap prices, and thus it is useful to incorporate the stochastic interest rate to form a more accurate model.

\section{Conclusion}

This paper investigates the pricing problem of variance and volatility swaps within the framework of stochastic volatility and stochastic interest rate. Upon successfully working out the expression of the forward characteristic function in a series form, accompanied by a radius of convergence, analytical pricing formulae for variance and volatility swaps are presented. The accuracy is numerically verified by the fact that swap prices from our formula and those from Monte Carlo simulation 


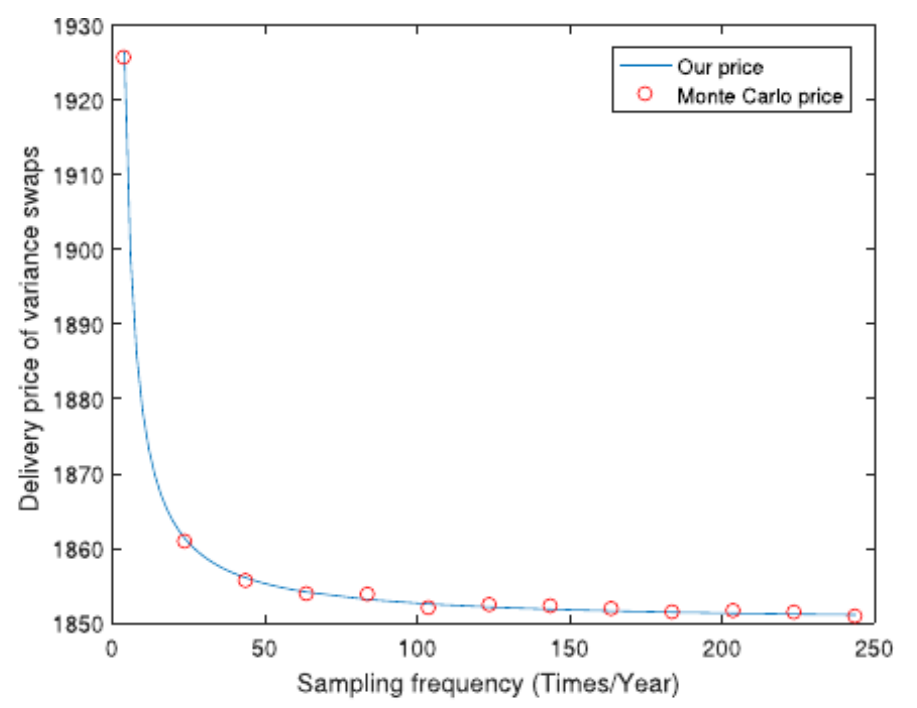

(a) Variance swap prices calculated from our formula and Monte Carlo simulation.

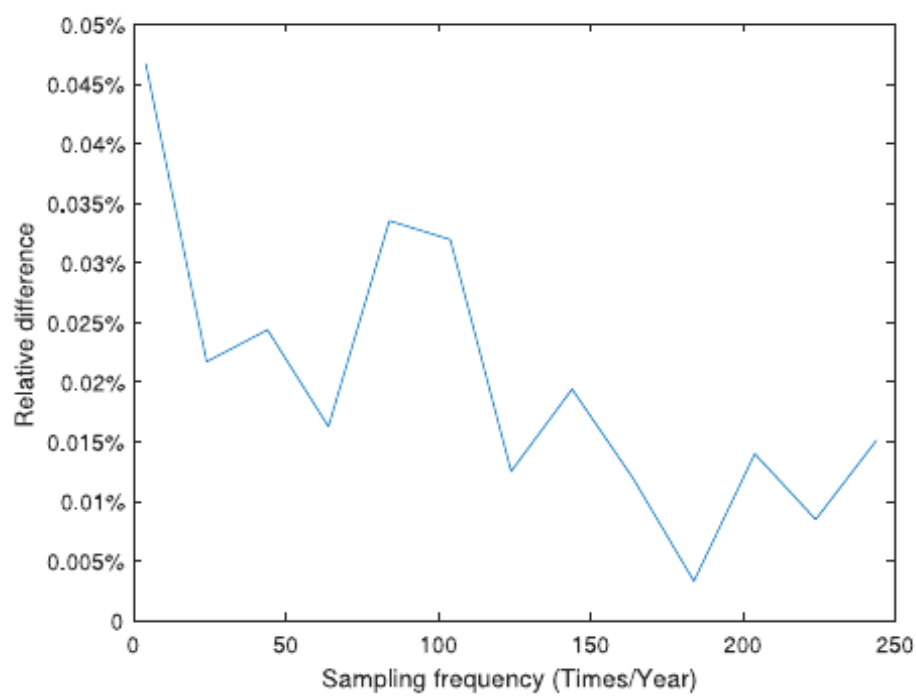

(b) Relative difference between our price and Monte Carlo price.

Fig. 2. Our price vs. Monte Carlo price.

model and those from the Heston model. Results show that stochastic interest rate has a significant impact on swap prices, and thus ignoring stochastic interest rate is not appropriate in practice.

\section{Appendix}

Here is the proof for Proposition 3.1.

Let us denote $h\left(\phi, t, T ; S_{s}, v_{s}, r_{s}, s\right)$ as the conditional forward characteristic function

$$
h\left(\phi, t, T ; S_{s}, v_{s}, r_{s}, s\right)=E^{Q^{T}}\left[e^{i \phi y_{t, T}} \mid S_{s}, v_{s}, r_{s}\right], s \in[t, T],
$$

then the PDE governing $h\left(\phi, t, T ; S_{s}, v_{s}, r_{s}, s\right)$ can be easily derived with the Feynman-Kac theorem

$$
\begin{aligned}
\frac{\partial h}{\partial \tau_{s}} & =\frac{1}{2} v \frac{\partial^{2} h}{\partial y^{2}}+\frac{1}{2} \sigma^{2} v \frac{\partial^{2} h}{\partial v^{2}}+\frac{1}{2} \eta^{2} r \frac{\partial^{2} h}{\partial r^{2}}+\rho \sigma v \frac{\partial^{2} h}{\partial y \partial v}+\left(r-\frac{1}{2} v\right) \frac{\partial h}{\partial y} \\
& +k(\theta-v) \frac{\partial h}{\partial v}+\left[\alpha \beta-\left(\alpha+B(s, T) \eta^{2}\right) r\right] \frac{\partial h}{\partial r},
\end{aligned}
$$

with $\tau_{s}=T-s$ and $\left.h\right|_{\tau_{\varepsilon}=0}=e^{j \phi y_{t, T}}$ as its initial condition. Following [13,35], $h\left(\phi, t, T ; S_{s}, v_{s}, r_{s}, s\right)$ is assumed to take the 


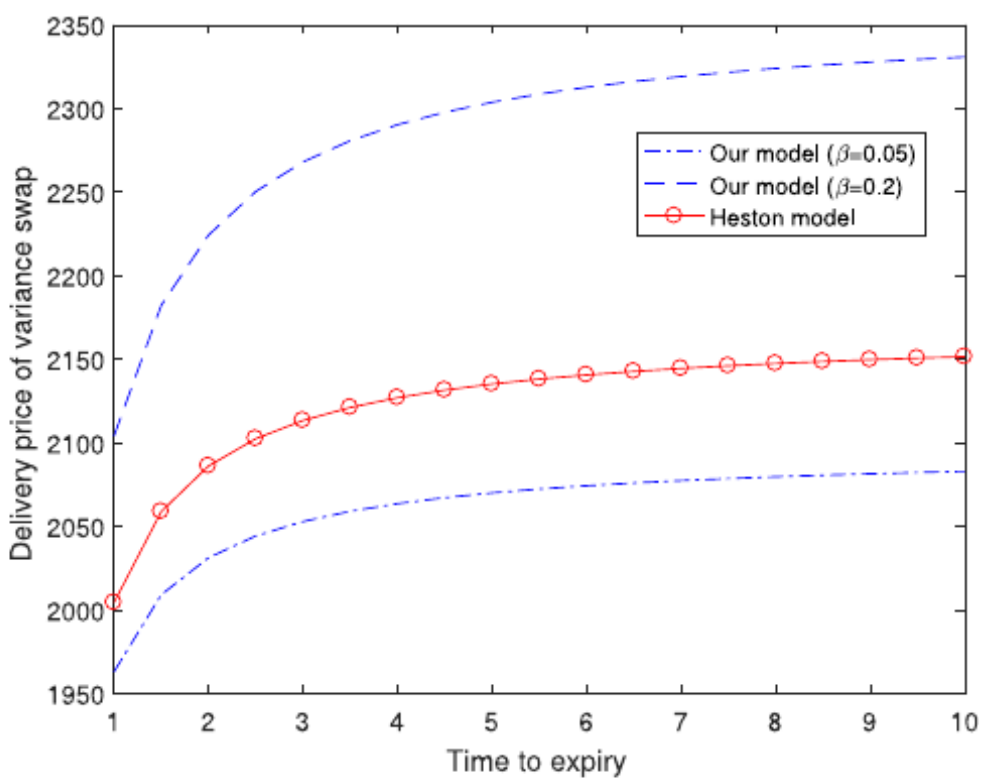

(a) Variance swap prices with respect to different time to expiry.

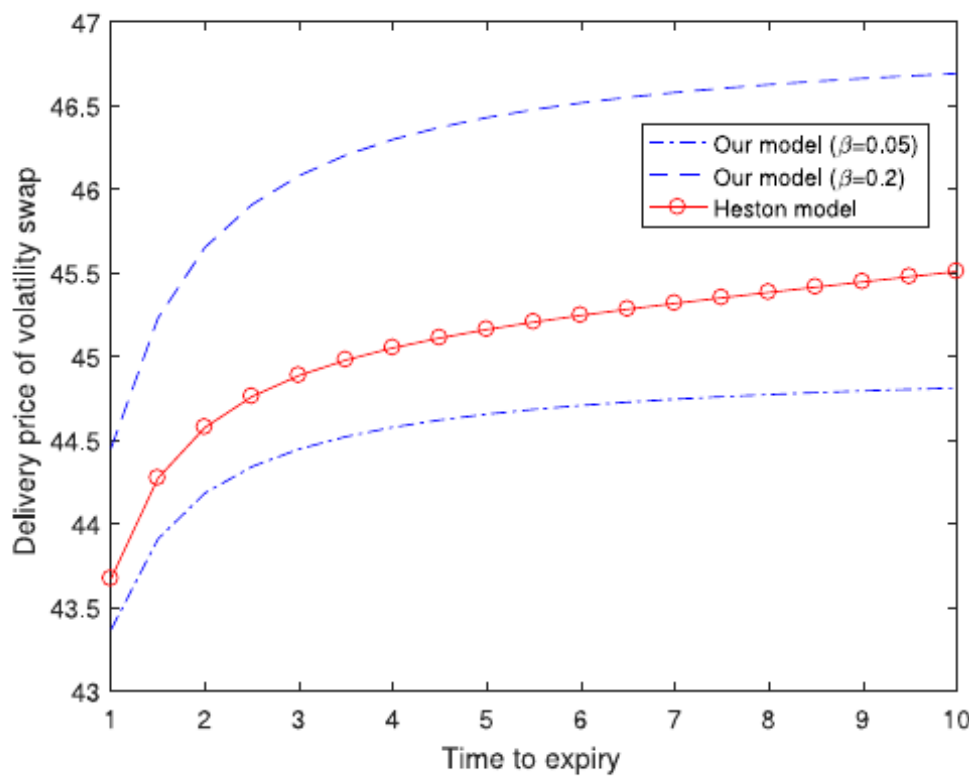

(b) Volatility swap prices with respect to different time to expiry.

Fig. 3. Our price vs. Heston price.

and the governing PDE can be reduced to the following three ODEs (ordinary differential equations)

$$
\begin{aligned}
& \frac{d D}{d \tau_{s}}=\frac{1}{2} \sigma^{2} D^{2}+(j \phi \rho \sigma-k) D-\frac{1}{2}\left(j \phi+\phi^{2}\right), \\
& \frac{d E}{d \tau_{s}}=\frac{1}{2} \eta^{2} E^{2}-\left[\alpha+B(s, T) \eta^{2}\right] E+j \phi, \\
& \frac{d C}{d \tau_{s}}=k \theta D+\alpha \beta E,
\end{aligned}
$$

with $D(\phi ; 0)=E(\phi ; 0)=C(\phi ; 0)=0$.

Obviously, the ODE governing $D\left(\phi ; \tau_{s}\right)$ is actually a Riccati equation with constant coefficients, which is not difficult to solve and thus details are omitted. A different case happens to the ODE governing $E\left(\phi ; \tau_{s}\right)$, as it is a Riccati equation with a time-dependent coefficient. In order to seek for an analvtical solution to this particular ODE, we make the transform of 
such that the original ODE can be reformulated as

$$
u^{\prime \prime}+\left[\alpha+B(s, T) \eta^{2}\right] u^{\prime}+\frac{1}{2} j \phi \eta^{2}=0 .
$$

In this way, we try to derive a series solution to Eq. (A.5) with respect to $\tau_{s}$

$$
u=\sum_{n=0}^{+\infty} a_{n} \tau_{s}^{n} .
$$

with which the ODE can be further simplified as

$$
\begin{aligned}
& {\left[2 m+(\alpha+m)\left(e^{m \tau_{s}}-1\right)\right] \sum_{n=0}^{+\infty}(n+1)(n+2) a_{n+2} \tau_{s}^{n}+\frac{1}{2} j \phi \eta^{2}\left[2 m+(\alpha+m)\left(e^{m \tau_{s}}-1\right)\right] \sum_{n=0}^{+\infty} a_{n} \tau_{s}^{n}} \\
& +\left\{\alpha\left[2 m+(\alpha+m)\left(e^{m \tau}-1\right)\right]+2 \eta^{2}\left(e^{m \tau}-1\right)\right\} \sum_{n=0}^{+\infty}(n+1) a_{n+1} \tau^{n}=0 .
\end{aligned}
$$

In order to derive all the coefficients $a_{n}$ in the series solution, $e^{m \tau_{s}}$ is expanded as $e^{m \tau_{s}}=\sum_{n=0}^{+\infty} c_{n} \tau_{s}^{n}$ so that the coefficients of $\tau_{s}^{n}$ can be put together. Considering the fact that Eq. (A.7) should hold for any $\tau_{s} \geq 0$, the coefficient of $\tau_{n}^{n}$ must equal to zero for all $n \geq 0$, which implies that

$$
\begin{aligned}
& 2 m(n+1)(n+2) a_{n+2}+2 \alpha m(n+1) a_{n+1}+j \phi \eta^{2} m a_{n}+(\alpha+m) \sum_{i=1}^{n}(n+2-i)(n+1-i) c_{i} a_{n+2-i} \\
& +\left(\alpha^{2}+\alpha m+2 \eta^{2}\right) \sum_{i=1}^{n}(n+1-i) c_{i} a_{n+1-i}+\frac{1}{2} j \phi \eta^{2}(\alpha+m) \sum_{i=1}^{n} c_{i} a_{n-i}=0 .
\end{aligned}
$$

From this, we can obtain a recurrence relationship for $a_{n}$ as

$$
a_{n+2}=-\frac{I}{2 m(n+1)(n+2)},
$$

where

$$
\begin{aligned}
I & =2 \alpha m(n+1) a_{n+1}+j \phi \eta^{2} m a_{n}+(\alpha+m) \sum_{i=1}^{n}(n+2-i)(n+1-i) c_{i} a_{n+2-i} \\
& +\left(\alpha^{2}+\alpha m+2 \eta^{2}\right) \sum_{i=1}^{n}(n+1-i) c_{i} a_{n+1-i}+\frac{1}{2} j \phi \eta^{2}(\alpha+m) \sum_{i=1}^{n} c_{i} a_{n-i} .
\end{aligned}
$$

However, the computation of $a_{2}$ from (A.9) requires both values of $a_{0}$ and $a_{1}$, while we only have $a_{1}=0$ from $E(\phi ; 0)=0$. Therefore, what we did is to introduce a new coefficient $\hat{a}_{n}=\frac{a_{n}}{a_{0}}$, such that $E\left(\phi ; \tau_{s}\right)$ in (A.4) can be rearranged as

$$
E=-\frac{2 \sum_{n=0}^{+\infty}(n+1) \hat{a}_{n+1} \tau^{n}}{\eta^{2} \sum_{n=0}^{+\infty} \hat{a}_{n} \tau^{n}},
$$

by dividing $a_{0}$ on both denominator and numerator. Here, $\hat{a}_{n}$ also has a recurrence relationship

$$
\hat{a}_{n+2}=-\frac{\hat{I}}{2 m(n+1)(n+2)},
$$

with $\hat{I}=\frac{I}{a_{0}}$. With $\hat{a}_{0}=1$ and $\hat{a}_{1}=0$ being easily found, all the coefficients $\hat{a}_{n}, n \geq 2$ can be derived through Eq. (A.11), which gives the expression of $E\left(\phi ; \tau_{s}\right)$, and thus the formula for $C(\phi ; \tau)$ can then be derived through direct integration. Clearly, we have now arrived at the analytical formula for the conditional forward characteristic function $h\left(\phi, t, T ; S_{s}, v_{s}, r_{s}, s\right)$ as all the unknown parts in (A.3) have been successfully derived. Setting $s=t$ in Eq. (A.3) yields

$$
h\left(\phi, t, T ; S_{t}, v_{t}, r_{t}, t\right)=E^{Q^{T}}\left[e^{j \phi y_{t, T}} \mid S_{t}, v_{t}, r_{t}\right]=e^{C(\phi ; \tau)+D(\phi ; \tau) v_{+} E(\phi ; \tau) r_{t}} .
$$

The disappearance of $y_{t, t}$ is simply due to $y_{t, t}=0$.

Since the derived formula is actually a series solution, it is necessary for us to check its convergence. The first and only 
to zero. Specifically, $\frac{1}{2} j \phi \eta^{2}$ in Eq. (A.5) is actually a constant and thus analytic in the entire complex domain. Moreover, $\alpha+B\left(\tau_{s}, T\right) \eta^{2}$ can be expressed as $\frac{F_{1}\left(\tau_{s}\right)}{F_{2}\left(\tau_{s}\right)}$, with

$$
\begin{aligned}
& F_{1}\left(\tau_{s}\right)=2 m \alpha+\left(\alpha^{2}+m \alpha+2 \eta^{2}\right)\left(e^{m \tau_{s}}-1\right), \\
& F_{2}\left(\tau_{s}\right)=2 m+(\alpha+m)\left(e^{m \tau_{s}}-1\right),
\end{aligned}
$$

both being analytic in the complex domain, and thus all the singularities can be obtained if we set $F_{2}\left(\tau_{s}\right)=0$. As a result, all singularities are

$$
l_{k}=\frac{1}{m} \ln \left(\frac{m-\alpha}{m+\alpha}\right)+j \frac{(2 k+1) \pi}{m}, k=0,1,2 \ldots
$$

Obviously, the nearest singularity to zero is $\frac{1}{m} \ln \left(\frac{m-\alpha}{m+\alpha}\right)+j \frac{\pi}{m}$, and thus the radius of convergence is at least $\frac{1}{m} \sqrt{\left[\ln \left(\frac{m-\alpha}{m+\alpha}\right)\right]^{2}+\pi^{2}}$. This has completed the proof.

\section{References}

[1] P. Carr, R. Lee, Realized volatility and variance: Options via swaps, Risk 20 (5) (2007) 76-83.

[2] P. Carr, R. Lee, Robust replication of volatility derivatives, in: PRMIA Award for Best Paper in Derivatives, MFA 2008 Annual Meeting, 2008.

[3] A. Grünbichler, F.A. Longstaff, Valuing futures and options on volatility, J. Banking Finance 20 (6) (1996) 985-1001.

[4] S. Howison, A. Rafailidis, H. Rasmussen, On the pricing and hedging of volatility derivatives, Appl. Math. Finance 11 (4) (2004) $317-346$.

[5] S. Heston, S. Nandi, Derivatives on volatility: some simple solutions based on observables, 2000.

[6] R.J. Elliott, T. Kuen Siu, L. Chan, Pricing volatility swaps under Heston's stochastic volatility model with regime switching, Appl. Math. Finance 14 (1) (2007) 41-62.

[7] T. Little, V. Pant, A finite difference method for the valuation of variance swaps, in: Quantitative Analysis in Financial Markets: Collected Papers of the New York University Mathematical Finance Seminar (Volume III), World Scientific, 2001, pp. 275-295.

[8] R.J. Elliott, G.-H. Lian, Pricing variance and volatility swaps in a stochastic volatility model with regime switching: discrete observations case, Quant. Finance 13 (5) (2013) 687-698.

[9] R. Farnoosh, A. Sobhani, H. Rezazadeh, M.H. Beheshti, Numerical method for discrete double barrier option pricing with time-dependent parameters, Comput. Math. Appl. 70 (8) (2015) 2006-2013.

[10] R. Farnoosh, A. Sobhani, M.H. Beheshti, Efficient and fast numerical method for pricing discrete double barrier option by projection method, Comput. Math. Appl. 73 (7) (2017) 1539-1545.

[11] G. Lian, S.-P. Zhu, R.J. Elliott, Z. Cui, Semi-analytical valuation for discrete barrier options under time-dependent lévy processes, J. Bank. Financ. 75 (2017) $167-183$.

[12] S.-P. Zhu, G.-H. Lian, A closed-form exact solution for pricing variance swaps with stochastic volatility, Math. Finance 21 (2) (2011) 233-256.

[13] S.L. Heston, A closed-form solution for options with stochastic volatility with applications to bond and currency options, Rev. Financ. Stud. 6 (2) (1993) $327-343$.

[14] S.-P. Zhu, G.-H. Lian, Analytically pricing volatility swaps under stochastic volatility, J. Comput. Appl. Math. 288 (2015) $332-340$.

[15] C. Bernard, Z. Cui, Prices and asymptotics for discrete variance swaps, Appl. Math. Finance 21 (2)(2014) 140-173.

[16] M. Broadie, A. Jain, The effect of jumps and discrete sampling on volatility and variance swaps, Int. J. Theor. Appl. Finance 11 (08) (2008) $761-797$.

[17] J.D. Hamilton, Analysis of time series subject to changes in regime, J. Econometrics 45 (1) (1990) 39-70.

[18] X.-J. He, S.-P. Zhu, How should a local regime-switching model be calibrated?, J. Econom. Dynam. Control 78 (2017) 149-163.

[19] X.-J. He, S.-P. Zhu, On full calibration of hybrid local volatility and regime-switching models, J. Futures Mark. 38 (5) (2018) $586-606$.

[20] X.-J. He, S.-P. Zhu, An analytical approximation formula for European option pricing under a new stochastic volatility model with regime-switching, J. Econom. Dynam. Control 71 (2016) 77-85.

[21] P. Carr, R. Lee, Volatility derivatives, Annu. Rev. Financ. Econ. 1 (1) (2009) 319-339.

[22] J. Hull, A. White, Pricing interest-rate-derivative securities, Rev. Financ. Stud. 3 (4) (1990) 573-592.

[23] L.A. Grzelak, C.W. Oosterlee, On the Heston model with stochastic interest rates, SIAM J. Financial Math. 2 (1) (2011) $255-286$.

[24] A. Medvedev, O. Scaillet, Pricing American options under stochastic volatility and stochastic interest rates, J. Financ. Econ. 98 (1) (2010) $145-159$.

[25] J.-H. Kim, J.-H. Yoon, S.-H. Yu, Multiscale stochastic volatility with the Hull-White rate of interest, J. Futures Mark. 34 (9) (2014) $819-837$.

[26] J. Cao, G. Lian, T.R.N. Roslan, Pricing variance swaps under stochastic volatility and stochastic interest rate, Appl. Math. Comput. 277 (2016) $72-81$.

[27] F. Black, M. Scholes, The pricing of options and corporate liabilities, J. Polit. Econ. (1973) 637-654.

[28] B. Dumas, J. Fleming, R.E. Whaley, Implied volatility functions: Empirical tests, J. Finance 53 (6) (1998) 2059-2106.

[29] M. Abudy, Y. Izhakian, Pricing stock options with stochastic interest rate, Int. J. Portfolio Anal. Manag. 1 (3) (2013) 250-277.

[30] D. Brigo, F. Mercurio, Interest Rate Models-Theory and Practice: With Smile, Inflation and Credit, Springer Science \& Business Media, 2007.

[31] E.M. Stein, J.C. Stein, Stock price distributions with stochastic volatility: An analytic approach, Rev. Financ. Stud. 4 (4) (1991) 727-752.

[32] D. Duffie, J. Pan, K. Singleton, Transform analysis and asset pricing for affine jump-diffusions, Econometrica 68 (6) (2000) $1343-1376$.

[33] D. Filipovic, Term-Structure Models. A Graduate Course., Springer, 2009.

[34] X.-J. He, S.-P. Zhu, A closed-form pricing formula for European options under the Heston model with stochastic interest rate, J. Comput. Appl. Math. 335 (2018) 323-333.

[35] X.-J. He, S.-P. Zhu, Pricing European options with stochastic volatility under the minimal entropy martingale measure, European J. Appl. Math. 27 (2) (2016) 233-247.

[36] C.M. Bender, S.A. Orszag, Advanced Mathematical Methods for Scientists and Engineers I, Springer Science \& Business Media, 1999. 